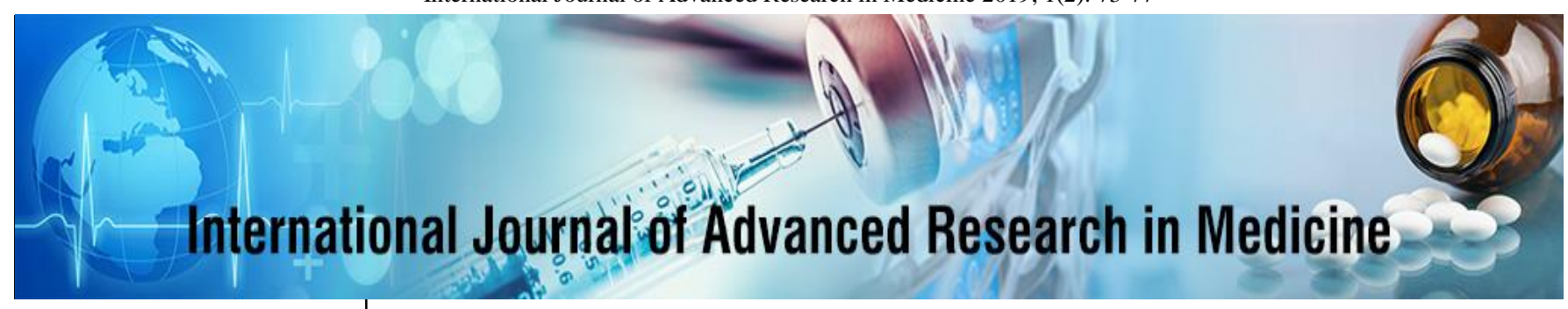

E-ISSN: 2706-9575

P-ISSN: 2706-9567

www.medicinepaper.net/ IJARM 2019; 1(2): 73-77

Received: 10-05-2019

Accepted: 12-06-2019

Rafal Ismail Abbas

Ministry of Health, Baghdad

Al-Russafa Health

Directorate, Madaen General

Hospital, Baghdad, Iraq

Athmar Jasim Mohammed Ministry of Health, Baghdad, Iraq

Khulood Abdul Wahab Jumaah

Ministry of Health - Baghdad Medical office - Al-Karkh, Karkh, Maternity Hospital, Baghdad, Iraq
Corresponding Author: Rafal Ismail Abbas Ministry of Health, Baghdad Al-Russafa Health Directorate, Madaen General Hospital, Baghdad, Iraq

\section{Diagnostic value of ultrasound in obstetrics and gynecology}

\author{
Rafal Ismail Abbas, Athmar Jasim Mohammed and Khulood Abdul \\ Wahab Jumaah
}

DOI: https://doi.org/10.22271/27069567.2019.v1.i2b.20

\begin{abstract}
The diagnosis of acute appendicitis in pregnant women often is difficult to make on the basis of clinical findings, and radiologic examination is limited because of the potentially hazardous effects of radiation.

This research is to assess the value of sonography in the diagnosis of acute appendicitis in pregnant women.

We obtained sonograms in (45) pregnant women with clinically suspected acute appendicitis. Our sonographic technique included graded-compression scanning. The left lateral decubitus position was used in the third trimester of gestation. The sonographic criterion for the diagnosis of acute appendicitis was visualization of an incompressible appendix with a maximal diameter greater than $(7 \mathrm{~mm})$.
\end{abstract}

Keywords: Ultrasound, hazardous, obstetrics \& gynecology, compression scanning

\section{Introduction}

We correlated the sonographic findings with the surgical findings in (22) cases and with the results of clinical follow-up in (23) cases. Sonography could not be used to make the diagnosis in three $(7 \%)$ of 45 patients because the size of the gravid uterus prevented use of the graded-compression technique.

These three patients were in the third trimester of pregnancy (greater than 35 weeks' gestation). Sonographic findings were used as a basis for diagnosis in 42 cases.

Acute appendicitis was diagnosed on the basis of sonograms in 16 patients, and in all but one of these patients, acute appendicitis was confirmed by surgical and pathologic findings.

In the 42 cases in which the imaging findings indicated the diagnosis, the overall sensitivity of sonography was $100 \%$, the specificity was $96 \%$, and the accuracy was $98 \%$. Our experience suggests that graded-compression sonography is a valuable procedure for detecting acute appendicitis in pregnant women despite technical difficulty in performing it during the third trimester of pregnancy.

Third trimester placental abruption complicates less than $1 \%$ of pregnancies but is associated with increased risk of preterm delivery and fetal death when it does occur. 1

The clinical diagnosis usually is based on bleeding, abdominal pain, and contractions, but sonography often is performed in an attempt to visualize the extent of subchorionic or retroplacental hematoma. When blood easily can drain through the cervix, however, no hematoma would be expected to be visualized. It is unclear how frequently sonography visualizes clots in cases of abruption and, if clots are visualized, whether management and outcome change as a result. Much of the literature on the subject is more than (20) years old. The purpose of this study was to estimate the sensitivity, specificity, positive predictive value (PPV), and negative predictive value (NPV) of sonography in the diagnosis of placental abruption and to determine, in women with clinical signs and symptoms of abruption, whether management and outcome are different when a hematoma is visualized compared with when no hematoma is apparent.

\section{Objective}

To determine the sensitivity and specificity of sonography for detection of placental abruption and whether sonographic results correlate with management or outcome. Methods. 


\section{Materials and Methods}

We performed breath-hold single-shot echo-planar diffusion-weighted MRI of the liver in 10 Healthy volunteers using conventional diffusion, conventional diffusion with parallel imaging, and diffusion tensor with parallel imaging sequences. TE values for the three sequences were 83,74 , and 63 , respectively.

Liver signal intensity was measured on all sequences and normalized to the SD of the measurement. Hepatic ADC was calculated by acquiring all sequences with $b$ values of 0 and $500 \mathrm{sec} / \mathrm{mm} 2$.

So, we identified (149) consecutive patients who underwent sonographic evaluation at 24 weeks' gestational age or longer for ruling out abruption or vaginal bleeding and who gave birth at Strong Memorial Hospital from 1994 to 1996. Scanning was performed primarily on Ultramark systems (Philips Ultrasound, Bothell, WA) by a group of 6 sonographers. Obstetric and neonatal data were obtained from the hospital perinatal database. We reviewed sonographic reports, pathologic reports, hospital charts, and a subset of images to confirm data validity and to determine whether sonographic findings were associated with management and outcome variables. A positive sonographic finding was defined as showing a subchorionic or retroplacental hematoma. Abruption at delivery was defined as a clinical diagnosis of placental abruption made at the time of delivery, whether by concurrent signs and symptoms or by examination of the placenta. Preterm delivery was delivery before 37 weeks' gestation, and low birth weight was defined as birth weight less than $2500 \mathrm{~g}$.

The sonographic sensitivity, specificity, PPV, and NPV were calculated. We used $\chi 2$, Fisher exact, Mann-Whitney U, and t-tests for comparisons $(P<.05)$. Linear and logistic regressions were used to determine independence of associations.

Odds ratios (ORs) were calculated from logistic coefficients. Analysis was done with Stat View 5.0 (SAS Institute Inc, Cary, NC) on a Macintosh G3 computer (Apple Computer, Inc, Cupertino, CA).

We identified (149) consecutive patients who underwent sonography at 24 weeks' gestational age or later for ruling out abruption or vaginal bleeding. Obstetric and neonatal data were obtained from the hospital perinatal database.

Sonographic results, pathologic reports, and hospital charts were reviewed. Sonographic sensitivity, specificity, and positive and negative predictive values were calculated, and regression was used to determine independence of associations. Results.

Of the (149) patients, 17 (11\%) had sonographic evidence of abruption, and $32(21 \%)$ had evidence of abruption at delivery. As the scan to delivery interval decreased, the positive predictive value increased and the negative predictive value decreased. of (55) patients who gave birth within (14) days of sonography, 8 (15\%) had scans consistent with abruption, and (29) (53\%) had abruption at delivery; the sensitivity, specificity, and positive and negative predictive values of sonography were $24 \%, 96 \%$, $88 \%$, and $53 \%$, respectively. Positive sonographic findings were univariately associated with (2-3) fold greater subsequent tocolysis, betamethasone use, duration of hospitalization, follow up sonograms, preterm delivery, low birth weight, and neonatal intensive care unit admission. All but low birth weight and neonatal intensive care unit admission remained independently significant after adjustment for gestational age $(P<.05)$.

\section{Results}

Of the 149 patients, $17(11 \%)$ had sonographic evidence of abruption, and $32(21 \%)$ had a clinical diagnosis of abruption at delivery (Table).

Including all patients, positive sonographic findings were univariately associated with approximately 2- to 3-fold greater subsequent tocolysis, betamethasone use, hospitalization-to-delivery duration, follow-up sonograms, preterm delivery, low birth weight, and neonatal intensive care unit admission (Tables 2 and).

When multiple linear and logistic regressions were used to control for gestational age at the time of scanning, all but low birth weight and neonatal intensive care unit admission were independently associated with positive sonographic findings.

When a diagnosis of abruption at delivery was added to the regressions as a dummy variable to try to separate the effect of the sonogram on management from the effect of the actual presence of abruption, betamethasone use still was independently associated with positive sonographic findings (or, 7.4; 95\% confidence interval [CI], 1.3-43.9; $\mathrm{P}=.03$ ) to a greater degree than abruption at delivery (OR, 3.5; 95\% CI, 1.2-10.4; $\mathrm{P}=.03$ ). Adjusting for abruption diagnosis at delivery, there was a trend toward independent association of sonographic results with tocolytic agent use and preterm delivery (OR for tocolysis, 2.9, 95\% CI, 0.89-9.4; $\mathrm{P}=.08$; OR for preterm delivery, $4.4 ; 95 \% \mathrm{CI}, 0.99-19.5 ; \mathrm{P}=.05$ ).

Table 1: Clinical Characteristics

\begin{tabular}{|c|c|c|c|}
\hline \multirow{2}{*}{ Characteristic } & \multicolumn{3}{|c|}{ Sonographic Findings } \\
\cline { 2 - 4 } & Positive, $\mathbf{n}=\mathbf{1 7 ( 1 1 \% )}$ & Negative, In = 132 (89\%) & p \\
\hline Graodity, median (range) & $4(14)$ & $3(1-11)$ & .31 \\
\hline Peary. median (range) & $1(0-4)$ & $1(0-7)$ & .18 \\
\hline Tobacco use. It (11) & $41(7)$ & $30(40)$ & .36 \\
\hline Cocaine use.\% (o) & $6(1)$ & $11(15)$ & .49 \\
\hline EGA at scan. vas medan (range) & $30(24-36)$ & $31(24.41)$ & .53 \\
\hline Scan to delivery, d. median (range) & $18(0-81)$ & $33(0-126)$ & .15 \\
\hline Hypertension. 1/4(n) & $0(0)$ & $6(8)$ & .30 \\
\hline Hydeamnen, 14(n) & $24(4)$ & $13(17)$ & .23 \\
\hline PROM, en (n) & $12(2)$ & $20(26)$ & .43 \\
\hline
\end{tabular}

For patients giving birth within 14 days of sonography, only tocolysis and betamethasone use were univariately associated with positive scan results. However, because of fewer patients in this group, power was less than $80 \%$ for 
most variables. As the scan-to-delivery interval decreased, PPV increased and NPV decreased (Table). Of 55 patients giving birth within 14 days of sonography, 8 (15\%) had positive scan results (consistent with abruption) and 29 (53\%) had clinical diagnoses of abruption at delivery; sensitivity, specificity, PPV, and NPV of sonography were $24 \%, 96 \%, 88 \%$, and $53 \%$, respectively.

For all scans and for those scans limited to within 14 days before delivery, neither placental location (anterior versus posterior) nor the stated indication for sonography (bleeding versus rule out abruption) significantly affected sensitivity, specificity, PPV, or NPV.

Sonography is not sensitive for detecting abruption, but when a clot is visualized on sonography, the PPV for abruption at delivery is high. The presence of blood in large enough volumes to be visible sonographically indicates retained hemorrhages that may be more likely to continue to manifest signs and symptoms.

In addition, large collections of blood would be expected to take longer to resorb or drain and are less likely to fully resolve by the time of delivery. The sooner delivery occurs after detection of such clots, therefore, the more often abruption will be clinically apparent.

The shorter the scan to delivery interval, the greater the PPV. When delivery occurred within 2 weeks of a positive sonographic finding, the diagnosis of placental abruption was confirmed in $100 \%$ of our cases.

Table 2: Obstetric Interventions \& Outcome

\begin{tabular}{|c|c|c|c|}
\hline & \multicolumn{2}{|c|}{ Sonographic Findings } & \\
\hline Intervention or Outcome & Positive & Negative & $\boldsymbol{P}$ \\
\hline Tocolysis, \%(n) & $71(12)$ & $34(45)$ & $.004 \bullet$ \\
\hline Iletamethasone, 66(n) & $87(13)$ & $32(36)$ & $<0001:$ \\
\hline Hospitalization to delivery, d, median (range) & $4(1-45)$ & $0(0-33)$ & $.0001:$ \\
\hline Follow-up sonogram, 96(n) & $73(11)$ & $37(41)$ & .008 \\
\hline Labor induction, 96(n) & $18(3)$ & $24(32)$ & .55 \\
\hline Cesarean delivery, \%(n) & $53(9)$ & $28(37)$ & .046 \\
\hline EGA at delivery, wk median (range) & $32(27-40)$ & $38(24-42)$ & .02 \\
\hline Postpartum hemorrhage, 96(n) & $18(3)$ & $4(5)$ & .02 \\
\hline
\end{tabular}

EGA indicates estimated gestational age.

Independently significant after adjustment for EGA at scanning (OR, 4.5; 95\% CI, 1.5-13.6; P =.008).

Independently significant after adjustment for tocolysis and EGA (OR, 15.3; 95\% CI, 3.1-75.2; P =.0008).

Independently significant after adjustment for EGA (regression coefficient, 4.4; $\mathrm{P}=.008$ ).

Not independently associated with scan result after adjustment for EGA.

Approximately $50 \%$ of women with clinical signs suggesting abruption but with negative sonographic findings have evidence of abruption at delivery. Blood having egress to the cervix may drain and thus may not collect under the chorion, so that no blood is visible during sonography. Such a patient still may be given a diagnosis of abruption at delivery based on intrapartum signs and symptoms of placental examination. Even if the placenta appears grossly normal, a diagnosis of abruption may follow from the classic appearance of vaginal bleeding, abdominal pain, and uterine hypertonicity.

As shown in Table (2), there were no significant differences in clinical characteristics between women with positive and women with negative sonographic findings. When a subchorionic or retroplacental hematoma was identified, however, management was more aggressive than when no hematoma was visualized. Women in whom sonography showed intrauterine hematomas more frequently received betamethasone and tocolytic agents, more commonly underwent follow-up sonograms, gave birth at earlier gestational ages, and had higher frequencies of postpartum hemorrhage. Did the positive sonographic findings themselves alter management, or were positive sonographic findings associated with more severe abruptions, the severity of which led to more aggressive management.

It's difficult to determine from the charts. After statistical adjustments, the sonographic result was more strongly associated with betamethasone use than was the clinical diagnosis of abruption itself, suggesting that for this intervention, the result did influence management. Because there is some multicollinearity between sonographic results and the diagnosis of abruption at delivery, adjusting for diagnosis at delivery might have masked some of the association of sonography with various interventions and outcomes.

Table 3: Neonatal Outcome

\begin{tabular}{|c|c|c|c|}
\hline \multirow{2}{*}{ Outcome } & \multicolumn{2}{|c|}{ Sonographic Findings } & \multirow{2}{*}{ p } \\
\cline { 2 - 3 } & Positive & Negative & \\
\hline Preterm delivery, 96(n) & $82(14)$ & $40(53)$ & .001 \\
\hline Birth weight, g, mean (50) & $2128(786)$ & $2682(936)$ & $.01 \bullet$ \\
\hline Low birth weight, \% (n) & $76(13)$ & $34(45)$ & $.0007 \bullet$ \\
\hline Apgar <7 at 5 min 96(n) & $6(1)$ & $5(6)$ & .81 \\
\hline WU admission, 96 (n) & $71(12)$ & $37(49)$ & $.008 \bullet$ \\
\hline
\end{tabular}

The Medline (1966-2001) and Embase (1980-2001) databases were searched for relevant studies, published in English. Only studies fulfilling predefined criteria were selected. An assessment of quality was made for each study, and data were then reanalyzed using likelihood ratios to determine the usefulness of the test.

Table 4: Accuracy of Sonography: Sonographic Result versus Clinical Abruption at Delivery

\begin{tabular}{|c|c|c|c|c|}
\hline \multirow{2}{*}{ Value } & \multicolumn{4}{|c|}{ Scan-to-Delivery Interval, wk } \\
\cline { 2 - 5 } & All & $\mathbf{3}$ & $\mathbf{2}$ & $\mathbf{1}$ \\
\hline $\mathrm{n}$ & 149 & 67 & 55 & 45 \\
\hline Sensitivity, 96 & 28 & 28 & 24 & 23 \\
\hline Specificity, 96 & 93 & 94 & 96 & 100 \\
\hline PPV, 96 & 53 & 82 & 88 & 100 \\
\hline NPV,46 & 83 & 59 & 53 & 49 \\
\hline
\end{tabular}


In summary, sonography is not sensitive for confirming placental abruption, although its PPV is high, especially when the scan to delivery interval is short. Given a positive sonographic result, obstetric management is more aggressive, and perinatal outcome is worse than when the sonographic result is normal.

Given this information, patients may be counseled more specifically regarding expected treatment and prognosis. Previous studies have related outcome to the sonographic appearance or to second-trimester scans, 35 but this study compared outcomes in groups with and without sonographic evidence of third-trimester abruption.

\section{Discussion}

Despite improvements in sonographic machines over the years, the diagnostic sensitivity for abruption has not improved in the past 2 decades. 2 Only 1 of every 9 sonograms obtained to rule out placental abruption revealed evidence of a subchorionic or retroplacental hematoma.

The test had a relatively low yield: sonographic findings for these indications usually are normal and are positive in only $25 \%$ of cases of placental abruption that are confirmed at delivery.

\section{Conclusions}

Sonography is not sensitive for detection of placental abruption, but a positive finding is associated with more aggressive management and worse neonatal outcome.

The normalized liver signal intensity was higher on diffusion tensor with parallel imaging and conventional diffusion with parallel imaging than on conventional diffusion without parallel imaging for a, b, value of 500 $\mathrm{sec} / \mathrm{mm} 2$ (13.0 and 10.1 vs 9.1 , respectively; $p<0.03)$ and for $a b$ value of $0 \mathrm{sec} / \mathrm{mm} 2$ (9.0 and 7.6 vs 6.9, respectively; without reaching a significant difference, $\mathrm{p}=0.12$ ).

Hepatic ADC was not significantly different between sequences $(\mathrm{p}=0.16)$.

Higher signal intensity can be obtained when using parallel imaging and diffusion tensor imaging during diffusionweighted MRI of the liver without compromising hepatic ADC measurement.

Presented at the 2004 annual meeting of the American Roentgen Ray Society, Miami Beach, FL. Partially supported by a grant from the Margulis Society, Department of Radiology, University of California, San Francisco.

\section{References}

1. Andersen B, Nielsen TF. Appendicitis in pregnancy: diagnosis, management, and complications. Acta Obstet Gynecol Sc and. 1999; 78:758-762.

2. Cunningham FG, McCubbin JH. Appendicitis complicating pregnancy. Obstet Gynecol. 1975; 45:415-420.

3. Moskovitz S, Gutman G, Lessing JB, Kupferminc MJ, Gamzu R. The significance of clinical signs and blood indices for the diagnosis of appendicitis during pregnancy. Gynecol Obstet Invest. 2003; 56:188-191.

4. Rioux M. Sonographic detection of the normal and abnormal appendix. AJR. 1992; 158:773-778.

5. Ames Castro M, Shipp TD, Castro EE, Ouzounian J, Rao P. The use of helical computed tomography in pregnancy for the diagnosis of acute appendicitis. Am J Obstet Gynecol. 2001; 184:954-957.
6. Incest L, Coskun A, Selcuk MB, Akan H, Sozubir S, Bernay $\mathrm{F}$ et al. Acute appendicitis: MR imaging and sonographic correlation. AJR. 1997; 168:669-674.

7. Rosai J. Appendix. In: Rosai J, ed. Rosai and Ackerman's surgical pathology, 8th ed. St. Louis, MO: Mosby, 1996, 711-716.

8. Fried AM. Hydronephrosis of pregnancy: ultrasonographic study and classification of asymptomatic women. Am J Obstet Gynecol. 1979; 135:1066-1070.

9. Popkin CA, Lopez PP, Cohn SM, Brown M, Lynn M. The incision of choice for pregnant women with appendicitis is through McBurney's point. Am J Surg. 2002; 183:20-22.

10. Zoetelief J, Geleijns J. Patient doses in spiral CT. Br J Radiol. 1998; 71:584-586.

11. International Commission on Radiological Protection. Pregnancy and irradiation. Annals of the ICRP. Stockholm, Sweden: Elsevier Scientific. 2000; 30:1-43. Publication 84.

12. Shellock FG, Kanal E. Policies, guidelines, and recommendations for MR imaging safety and patient treatment: SMRI safety committee. J Magn Reson Imaging. 1991; 1:97-101.

13. Murtz P, Flacke S, Traber F, van den Brink JS, Gieseke J, Schild HH et al. Abdomen: diffusion-weighted MR imaging with pulse-triggered single-shot sequences. Radiology. 2002; 224:258-264.

14. Willinek WA, Gieseke J, von Falkenhausen M, Neuen B, Schild HH, Kuhl CK et al. Sensitivity encoding for fast MR imaging of the brain in patients with stroke. Radiology. 2003; 228:669-675.

15. Bammer R, Keeling SL, Augustin M. Improved diffusion-weighted single-shot echo-planar imaging (EPI) in stroke using sensitivity encoding (SENSE). Magn Reson Med. 2001; 46:548-554.

16. Griswold MA, Jakob PM, Chen Q. Resolution enhancement in single-shot imaging using simultaneous acquisition of spatial harmonics (SMASH). Magn Reson Med. 1999; 41:1236-1245.

17. Jaermann T, Crelier G, Pruessmann KP. SENSE-DTI at 3 T. Magn Reson Med. 2004; 51:230-236.

18. Carlson JW, Minemura $\mathrm{T}$. Imaging time reduction through multiple receiver coil data acquisition and image reconstruction. Magn Reson Med. 1993; 29:681687.

19. Weiger M, Pruessmann KP, Kassner A. Contrastenhanced 3D MRA using SENSE. J Magn Reson Imaging. 2000; 12:671-677.

20. Weiger M, Pruessmann KP, Boesiger P. Cardiac realtime imaging using SENSE: SEN Sitivity Encoding scheme. Magn Reson Med. 2000; 43:177-184.

21. Golay X, Pruessmann KP, Weiger M. PRESTOSENSE: an ultrafast whole-brain fMRI technique. Magn Reson Med. 2000; 43:779-786.

22. Yoshioka H, Takahashi N, Yamaguchi M, Lou D, Saida Y, Itai Y et al. Double arterial phase dynamic MRI with sensitivity encoding (SENSE) for hypervascular hepatocellular carcinomas. J Magn Reson Imaging. 2002; 16:259-266.

23. Gosse C, Fahrmeir L, Putz B, Auer LM, Auer DP. Fiber tracking from DTI using linear state-space models: detectability of the pyramidal tract. Neuroimage. 2002; 16:378-388. 
24. Le Bihan D, Mangin JF, Poupon C. Diffusion tensor imaging: concepts and applications. J Magn Reson Imaging. 2001; 13:534-546.

25. Tench CR, Morgan PS, Wilson M, Blumhardt LD. White matter mapping using diffusion tensor MRI. Magn Reson Med. 2002; 47:967-972.

26. Le Bihan D. Looking into the functional architecture of the brain with diffusion MRI. Nat Rev Neurosci. 2003; 4:469-480.

27. Ries M, Jones RA, Basseau F, Moonen CT, Grenier N. Diffusion tensor MRI of the human kidney. J Magn Reson Imaging. 2001; 14:42-49. 